\title{
FACTORES HEDÓNICOS Y MULTICULTURALES QUE MEJORAN LA EXPERIENCIA DE USUARIO EN EL DISEÑO DE PRODUCTOS
}

\author{
José-Luis González-Sánchez y Rosa-María Gil-Iranzo
}
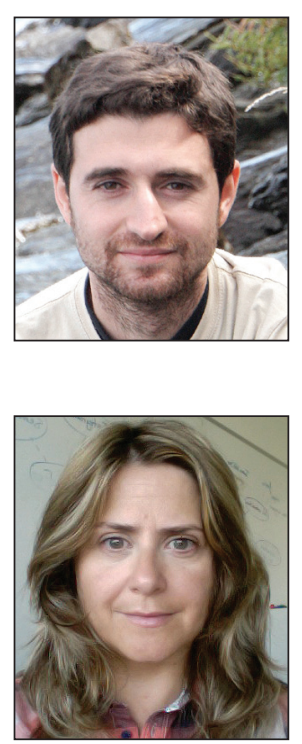

José-Luis González-Sánchez es doctor y profesor del Depto. de Informática e Ingeniería Industrial en la Escuela Politécnica Superior de la Univ. de Lleida (UdL). Imparte docencia sobre ingeniería del software en los estudios de grado en informática y en diferentes masters oficiales de la $U d L$ y de la Univ. de La Laguna. Es miembro de la Asoc. Española de Interacción Persona Ordenador y de la Academia de Artes y Ciencias Interactivas. Investiga en la caracterización y evaluación de la experiencia del usuario en sistemas interactivos.

http://orcid.org/0000-0002-0777-8427

joseluisgs@diei.udl.cat

Rosa-María Gil-Iranzo, doctora en informática, es profesora en el Departamento de Informática e Ingeniería Industrial de la Universitat de Lleida. Imparte docencia en estudios de grado en informática y en el master de interacción persona-ordenador de la Universitat de Lleida. Su trabajo se centra en el campo de la arquitectura de la información, web semántica y dentro de la experiencia de usuario en el diseño multicultural, patrones web y modelos mentales de usuarios. http://orcid.org/0000-0001-8981-6273

rgil@diei.udl.cat

Universitat de Lleida, Escuela Politécnica Superior Depto. de Informática e Ingeniería Industrial Jaume II, 69. 18071 Lleida, España

\section{Resumen}

En un mundo donde los sistemas y mecanismos de comunicación e información son cada vez mayores, una correcta expe riencia de usuario se convierte en un factor determinante para asegurar el éxito de un producto en mercados cada día más saturados y competitivos. Asegurar el éxito de un producto, como una página web o un sistema móvil, no es algo trivial y pueden ser muchos factores los que determinen si la experiencia es correcta o no para un determinado sector de usuarios. Se analiza mediante ejemplos reales el concepto de experiencia de usuario, profundizando en distintos factores decisivos en el éxito final de un producto que influyen en el tratamiento y diseño de los sistemas interactivos y de información y mostrando cómo se ven respaldados por los distintos estándares en busca de una mejor experiencia del usuario.

\section{Palabras clave}

Experiencia de usuario, Multiculturalidad, Diseño emocional, Calidad en uso, Gamificación, Ludificación.

Title: Hedonic and multicultural factors in product design that improve the user experience

\section{Abstract}

In a world where the use of electronic communication and information systems is constantly expanding, a good user experience becomes a decisive factor for the success of a product in increasingly saturated and competitive markets. Ensuring the success of a product such as a website or a mobile application is not trivial as plenty of factors may have an influence on determining the adequacy of the experience for a given group of users. This paper uses real examples to study the concept of user experience, dealing in depth with the different factors which influence the ultimate success of a product, as well as how they must be taken into account while designing and managing interactive information systems. We also discuss the way they are supported by the different standards to improve user experience.

\section{Keywords}

User experience, Cross-cultural, Emotional design, Quality in use, Gamification.

González-Sánchez, José-Luis; Gil-Iranzo, Rosa-María (2013). "Factores hedónicos y multiculturales que mejoran la experiencia de usuario en el diseño de productos”. El profesional de la información, enero-febrero, v. 22, n. 1, pp. 26-35.

http://dx.doi.org/10.3145/epi.2013.ene.04 


\section{Introducción}

En la percepción que un usuario tiene de un sistema de información interactivo influyen aspectos internos y externos al mismo: sociales, subjetivos del usuario, entorno de uso, campañas de marketing, servicios de atención al cliente, comentarios o recomendaciones. Todos ellos determinan la correcta experiencia interactiva o experiencia del usuario (UX) (Garret, 2010; Law et al., 2008).

Los constantes avances tecnológicos hacen que cada vez se estén considerando más factores y criterios en su diseño, desarrollo y evaluación para mejorar su calidad. Desde el punto de vista de la interacción y arquitectura de la información, el concepto que desde hace tiempo viene utilizándose para valorar parte de dicha experiencia es el de la usabilidad, pero ésta no es suficiente para caracterizarla y ver su grado de adecuación al posible grupo de usuarios al que va orientado el sistema. Factores subjetivos y hedónicos como el contexto cultural, colores, impacto emocional o el modelo mental que los usuarios tienen del sistema influyen en que la experiencia sea más positiva o negativa en un contexto de uso determinado.

El objetivo principal de este trabajo es acercar y debatir el grado de influencia que dichos factores subjetivos y hedónicos tienen a la hora de diseñar un sistema con el fin de generar la mejor experiencia interactiva posible, ilustrando la importancia de algunos de los factores más destacados a través de ejemplos. En primer lugar, se analiza el concepto de experiencia de usuario, cómo se caracteriza y cómo está respaldada según los últimos estándares internacionales. Seguidamente se profundiza sobre qué variantes y factores influyen en mayor y menor medida en la experiencia interactiva. Se mostrarán ejemplos de su aplicación por parte de empresas reconocidas en sus diferentes ámbitos. Debido a la heterogeneidad del contenido y de cada una de las propiedades se muestran ejemplos a lo largo de este artículo para ilustrar cada uno dichos factores o propiedades de la manera más destacada y con la menor interferencia posible. Finalmente se analiza el contexto actual y las ideas

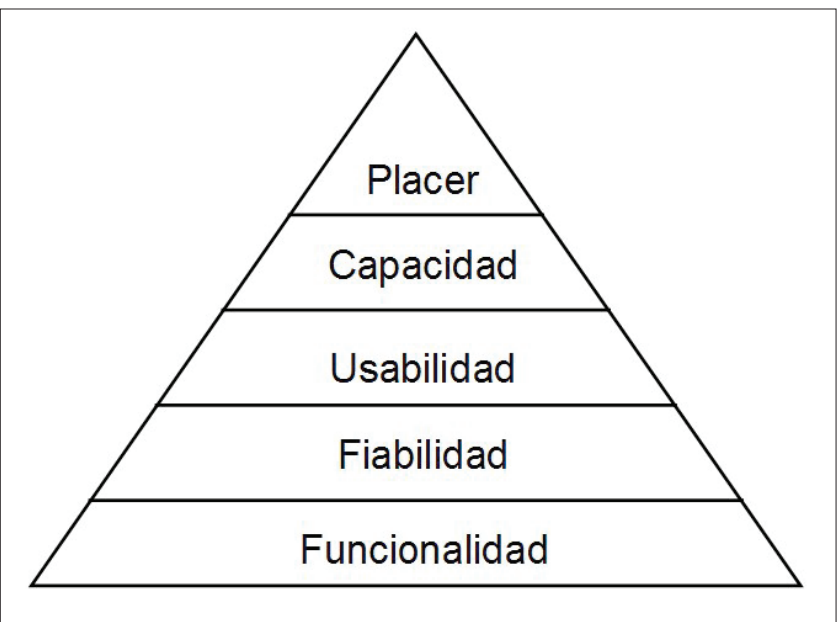

Figura 1. Pirámide de Maslow para la jerarquía de diseño de necesidades del usuario

http://www.smashingmagazine.com/2010/04/26/designing-for-ahierarchy-of-needs futuras que se desprenden de los resultados mostrados en este trabajo.

\section{Experiencia de usuario, usabilidad y calidad en uso}

El estudio de la experiencia de usuario (UX) tiene como objetivo conocer las emociones y sensaciones que un usuario obtiene como respuesta al interactuar con un sistema e incluye aspectos tanto subjetivos como culturales, y de familiaridad del usuario con otros productos de la marca o similares (Law; Van-Schaik, 2010; Law et al., 2009; HassanMontero; Martín-Fernández, 2005). Es decir, la UX complementa la información objetiva de la realización de una tarea con información subjetiva sentida a la hora de realizarla. Siendo la UX dinámica y dependiente del contexto, su análisis ayuda a mejorar la aceptación de los usuarios, ganar su fidelidad y mejorar el retorno económico del desarrollo del producto. La UX se define formalmente como "la percepción de una persona y las respuestas subjetivas de ésta como resultado de la utilización y/o el uso de un producto, sistema o servicio" (ISO/IEC 9241-210: 2010). Por lo tanto a la hora de asegurar una buena experiencia de usuario es recomendable conocer al conjunto de usuarios que harán uso del producto con el objetivo de intentar experiencias y sensaciones posibles durante el proceso interactivo.

\section{Una correcta experiencia de usuario es determinante para asegurar el éxito de un producto en mercados cada día más saturados y competitivos}

Se considera la usabilidad (Nielsen, 1993; Mayhew, 1999) como uno de los aspectos clave a la hora de evaluar la UX, de hecho, su atributo de satisfacción ha sido durante mucho tiempo el más utilizado para este aspecto. Pero al evaluar la UX debemos diferenciar los aspectos pragmáticos o funcionales (¿qué y para qué realizamos una tarea?) de los hedónicos desprendidos del proceso de uso del mismo (¿qué sentimos al realizar la tarea?), pues influyen en mayor o menor medida en la aceptación de un producto. La usabilidad asegura la calidad del producto software, pero que debe ser completado para cubrir aspectos relacionados con una completa experiencia (Baeza-Yates; Rivera-Loaiza; VelascoMartín, 2004). Los enfoques tradicionales de evaluación de la satisfacción (como la usabilidad) analizan generalmente la percepción de "satisfacción" según la eficacia y eficiencia, de modo que si los usuarios perciben como eficaz y eficiente el uso del producto, se da por hecho que están satisfechos (Hassenzahl, 2003). Pero hay aspectos de la UX, como la diversión, placer o influencia sociocultural, que contribuyen de manera significativa a la satisfacción del usuario respecto con el producto (Cockton, 2008). Estos factores han llegado a formar parte del nuevo estándar de la calidad en uso ISO/ IEC 25010 (ISO/IEC 25010:2011).

Dentro de la experiencia de usuario, a nivel de estándares se ha hecho énfasis en formalizar aspectos pragmáticos 


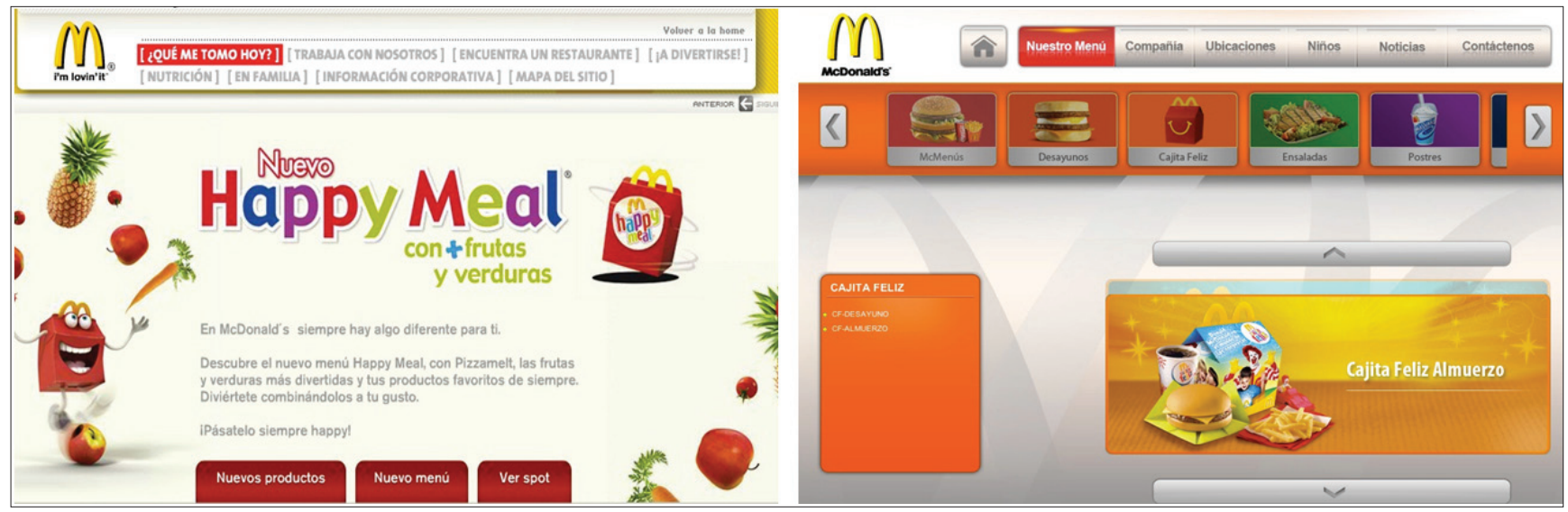

Figura 2. Diferencias en contextualización en la web de McDonald's para España (izquierda) y Guatemala (derecha)

como la usabilidad, como ISO 9241-11:1998 o ISO/IEC 91261:1991 o su revisión ISO/IEC 9126-1:2001. Debido a la rapidez de cambios y tendencias de mercado y a la dificultad de los estándares de actualizarse continuamente, la UX se vio regida por distintas normas de facto que identificaban los aspectos hedónicos (tendencias de colores, estilos de diseño según el mercado, etc.). Pero los esfuerzos promovidos de distintas compañías, investigadores, expertos y profesionales del sector en pos de un consenso y de promover una norma común ha derivado en la aparición de dos nuevos estándares ISO centrados en la definición o contextualización de factores de la experiencia interactiva ligados al concepto de calidad (Bevan, 1999; Bevan, 2000; Cockton, 2008; Law; Van-Schaik, 2010). La primera norma a destacar es la ISO/ IEC 9241-210, cuya actualización añade una nueva cláusula que define el concepto de UX y las diferentes facetas para su caracterización (hedónicas y pragmáticas) (ISO/IEC 9241210:2010). Además, el estándar de calidad en uso ISO/IEC 25010 se centra en definir los atributos y propiedades necesarios para asegurar un cierto grado de calidad en el proceso de uso de un producto software. Del primero ya se ha hablado en párrafos anteriores, del segundo hay que mencionar los factores medibles:

- Efectividad: exactitud e integridad con que los usuarios alcanzan objetivos específicos o realizan una tarea.
- Eficiencia: recursos invertidos en relación con la exactitud e integridad con que los usuarios realizan una tarea.

Satisfacción: grado en que las necesidades del usuario están satisfechas cuando un producto o sistema se utiliza en un contexto de uso específico e incluye las actitudes hacia el uso del producto.

- Libertad de riesgo: grado en que un producto o sistema reduce el riesgo potencial a nivel económico, humano, salud o del medio ambiente.

Cobertura del contexto: grado en que un producto o sistema puede ser utilizado con eficacia, eficiencia, libre de riesgo y con satisfacción en el contexto de uso específico (adecuación al contexto) y en aquellos más allá de los inicialmente identificados (flexibilidad en uso).

Algunos autores (Krug, 2005; Law, 2011, Vermeeren et al., 2010) discuten los métodos y necesidades actuales a tener en cuenta en la caracterización de experiencia interactiva en sistemas software y webs destacando la importancia de la respuesta emocional (satisfacción), atractividad o uso de colores y las restricciones debidas a la influencia sociocultural (adecuación al contexto / flexibilidad en uso) atendiendo a los usuarios a los que van destinados. Es por ello, que los métodos de diseño y evaluación deben enriquecerse con dichos factores para conseguir información más completa de la calidad de la experiencia en el proceso de interacción
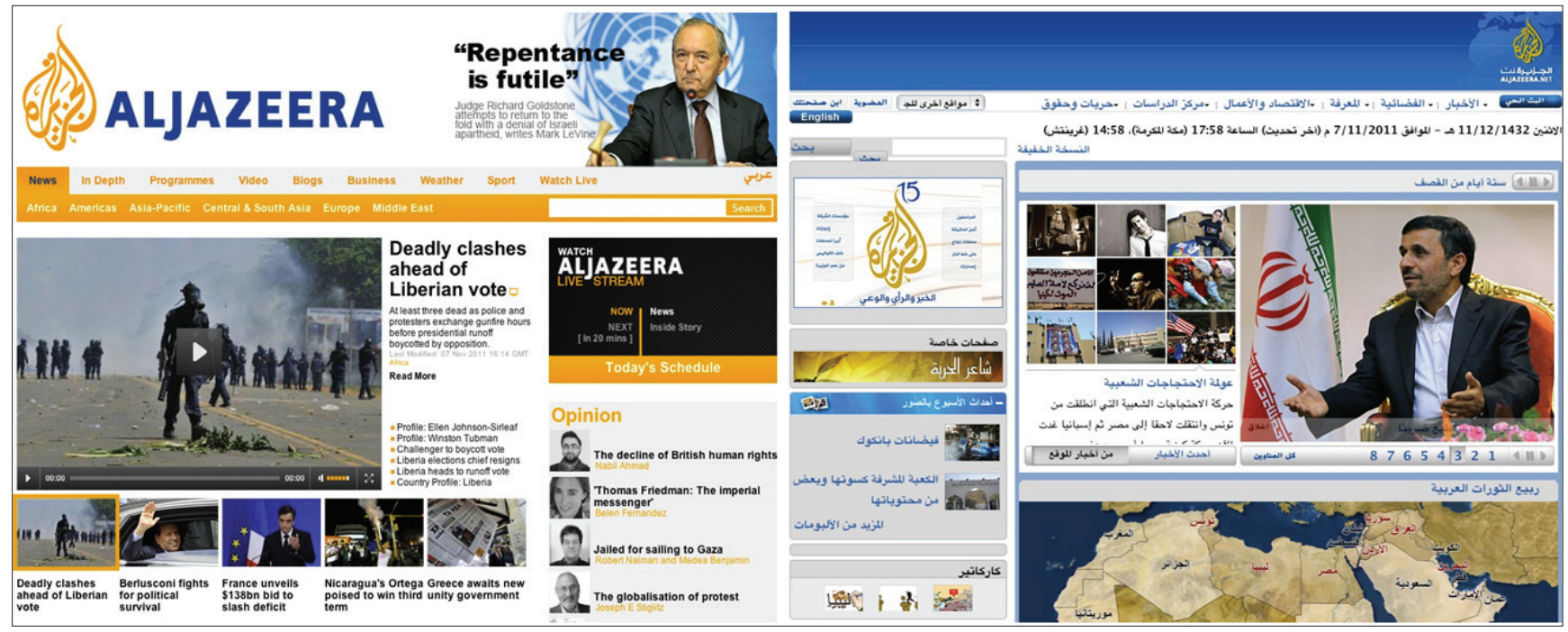

Figura 3. Disposición de menús e imágenes de Al Jazeera para usuarios no árabes y para usuarios árabes 
usuario-sistema. En la jerarquía de la Pirámide de Maslow (Maslow, 1943), modificada para el diseño de las necesidades del usuario, se muestran los elementos influyentes en la experiencia interactiva que van más allá de la usabilidad (figura 1). En el nivel inferior está la funcionalidad (el sistema funciona, soluciona las necesidades básicas y el diseño apenas se percibe), encima se sitúa la fiabilidad (funcionamiento estable y coherente), más arriba se encuentra la usabilidad (facilidad de uso, el diseño -que se muestra como un valor moderado- admite que el usuario se equivoque $y$ cometa fallos), en la capa superior hallamos la capacidad, el dominio o la habilidad para realizar el objetivo (empoderar al usuario para hacer más y mejores cosas) y finalmente en la cumbre está la creatividad y el placer (belleza estética, interactividad innovadora, se disfruta el buen diseño).

\section{Contexto cultural y experiencia de usuario}

La influencia sociocultural en el usuario puede provocar grandes diferencias en el desarrollo de productos. Hofstede (2005) considera la cultura como una programación mental, pues cada persona tiene en su interior modelos de pensamiento, sentimiento y actuación que actúan como "software mental".

Las personas se diferencian por aspectos culturales entre sociedades y por características internas. Estos meta-modelos culturales (LLC, 2009), que representan la influencia sociocultural, provocan que "Ios diseños de los sistemas software y de información sigan unas ciertas reglas o patrones dependiendo del grupo de usuarios al que va dirigido, con el fin de mejorar la experiencia en el proceso de uso y asegurar que la elección de metáforas, colores, organización de la información sea la adecuada para la población a la que va destinada el producto" (Collazos; Gil, 2011; Andreu; Marcos, 2011). El contexto o multiculturalidad influye en la experiencia final y va ligado al concepto de "flexibilidad" dentro de la "cobertura del contexto" del estándar ISO/IEC 25010. A su vez nos asegura el atributo de "libertad de riesgos", al no utilizar metáforas o información ofensiva para los usuarios o que incumplan algún aspecto legal en países determinados.
Dada la heterogeneidad del contenido, se ofrece con cada factor un ejemplo que ilustra su apreciación e importancia para el diseño del producto interactivo:

\section{Traducción y acercamiento}

Si un mensaje está escrito en otro idioma, debe ser traducido y contextualizado. Sin embargo, existen variantes del lenguaje que provocan divergencias en la experiencia. Las compañías internacionales tienden a contextualizar sus eslóganes publicitarios según su audiencia y realidades sociales. Por ejemplo, la cadena McDonald's usa el termino Happy Meal o I'm Lovin' it en países donde el grado de conocimiento de inglés es elevado o donde el marketing hacia productos anglosajones no es negativo, como puede ser el caso de España. Pero en países como Guatemala dichos mensajes son traducidos: Cajita Feliz o Me encanta. Esta realidad social ha provocado que en las respectivas webs se presente el mismo concepto de dos maneras diferentes. Este ejemplo, ilustra la importancia de la traducción/contextualización para mejorar la experiencia del usuario final (figura 2).

Aspectos de la UX como diversión, placer, influencia sociocultural, contribuyen a la satisfacción del usuario con el producto

\section{Dirección y simetría de la información}

Existen países/lenguas cuya escritura/lectura es realizada de derecha a izquierda y en ciertos países asiáticos es realizada de arriba abajo; en la cultura occidental, la lectura es de izquierda a derecha y de la parte superior a la inferior. Este hecho provoca que la disposición de la información influya en su comprensión. Algunos casos que muestran la importancia de la disposición de la información los tenemos accediendo al sitio web de la cadena de comunicación $A l$ Jazeera. En ella, el logotipo, menús y marquesinas están
En este artículo se ejemplifican evidencias mostradas por algunos de los patrones sociológicos, culturales y antropológicos más conocidos propuestos por Hofstede (2005), mostrando cómo aspectos culturales se plasman en su diseño con el objetivo de conseguir una correcta experiencia interactiva. Las recomendaciones sobre factores hedónicos donde se relacionan elementos multiculturales y el diseño web tenidos en cuenta en este trabajo son: traducción, dirección y simetría, color, individualismo / colectivismo, género, y arquitectura de la información.

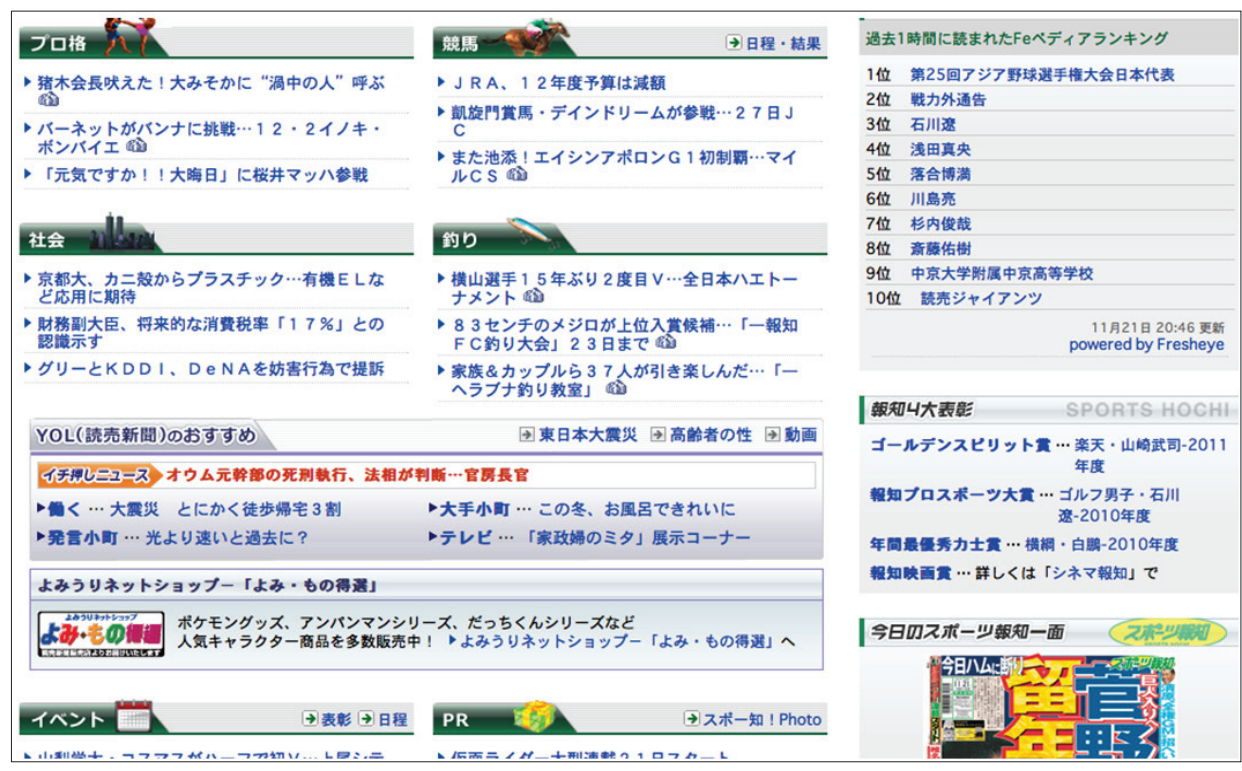

Figura 4. Utilización de páginas con columnas y escritura horizontal en uno de los cinco diarios japoneses con más ejemplares vendidos (Hochi Yomiuri, http://hochi.yomiuri.co.jp) 

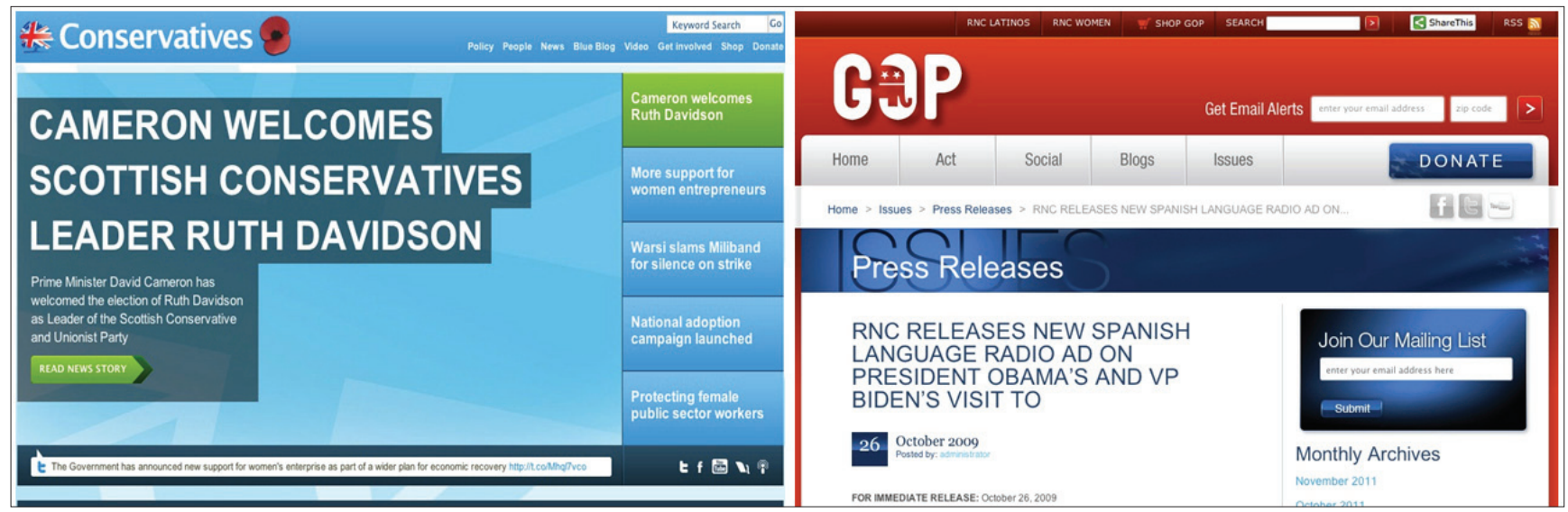

Figura 5. Uso del color para representar conceptos políticamente similares: el color azul en el Reino Unido (izquierda) y el partido ideológicamente similar usando el color rojo en los Estados Unidos (derecha)

adaptadas a los usuarios, respetando la disposición de los elementos ("adecuación al contexto" y "flexibilidad en uso") según sea la procedencia del usuario que entra a la web (figura 3). La simetría es un factor clave a la hora de aplicar patrones de diseño web.

Por otro lado, en ciertos países asiáticos, donde la lectura es en dirección vertical, las páginas webs empezaron a estructurarse en columnas muy largas, debido al tipo de su escritura y lectura y del procesamiento cognitivo de este tipo de usuarios. Pero la influencia de la cultura occidental (alfabeto, números arábigos y fórmulas matemáticas), y algunas lenguas como la japonesa, donde cada "kanji" y "kana" tiene su propio significado y estos pueden organizarse vertical $u$ horizontalmente sin perder su sentido, han provocado que el diseño web se estructure generalmente de forma horizontal, siguiendo el patrón usado en libros modernos. Además han hecho que los usuarios desarrollen un modelo cognitivo que permite procesar la información mostrada tanto de manera vertical como horizontal (la más común hoy en día) (Abramson et al., 1993; Savvas; El-Kot; Sadler-Smith, 2001; Allinson; Hayes, 2000; Shen; Woolley; Prior, 2006; Hofstade, 2005) (figura 4).

\section{Colores apropiados}

Los colores son identificativos y forman parte de la experiencia de uso provocando reacciones opuestas según sean utilizados. Un ejemplo lo tenemos con el rojo, que en países occidentales simboliza la excitación, utilizándose para llamar la atención o indicar situaciones de peligro y en culturas asiáticas, como la china o tailandesa, simboliza la buena suerte, la belleza o lo positivo (McCandless, 2010; Sharpe, 1974; Birren, 1978). Podemos ver en la figura 5 la importancia del color y su influencia en el mensaje político según el país haga uso de ellos y como consecuencia su protagonismo en el diseño de la web.

http://www.informationisbeautiful.net/visualizations/ colours-in-cultures

\section{Individualismo / colectivismo}

Las imágenes y metáforas gráficas deben ser las adecuadas para la población a la que va dirigido el producto. Mientras que en los países anglosajones los productos se orientan hacia la libertad y poder individual (motivación ante el desarrollo personal), en los países latinos y asiáticos es más adecuado apostar por el concepto de grupo y la parte social (motivación ante el desarrollo colectivo) (Hofstede, 2005). Estas bases sociológicas se muestran en la elección de ico nos o mensajes en las páginas iniciales de determinadas empresas y en sus campañas de marketing (figura 6).

\section{Adecuación del género}

Los estereotipos de masculinidad y feminidad referidos a los roles de género son ampliamente utilizados, pero la percepción de lo que es sexista cambia dependiendo de la población. Lo masculino suele ligarse a la asertividad, la competencia y la dureza, mientras la feminidad se une a la ternura, las labores del hogar y el cuidado de las personas (Hofstede, 1998; 2005). En la web tienden a propagarse aspectos estereotipados sociológicamente aceptados por la comunidad según se orienten al sector masculino o femenino. Depen-

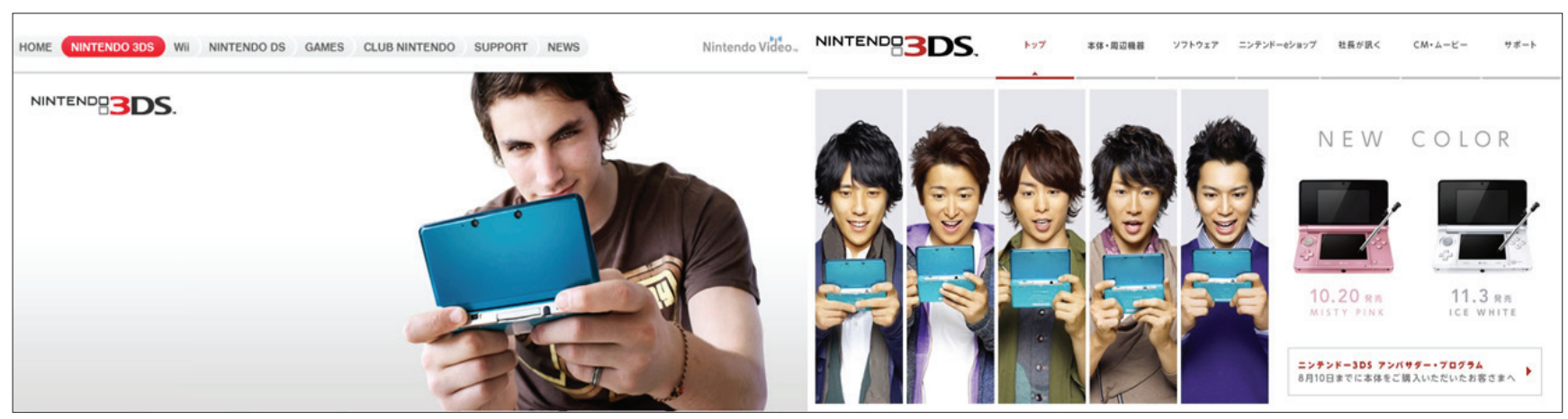

Figura 6. Metáforas del uso del individualismo / colectivismo en la web de Nintendo 3DS de Reino Unido (izquierda) y Japón (derecha) 


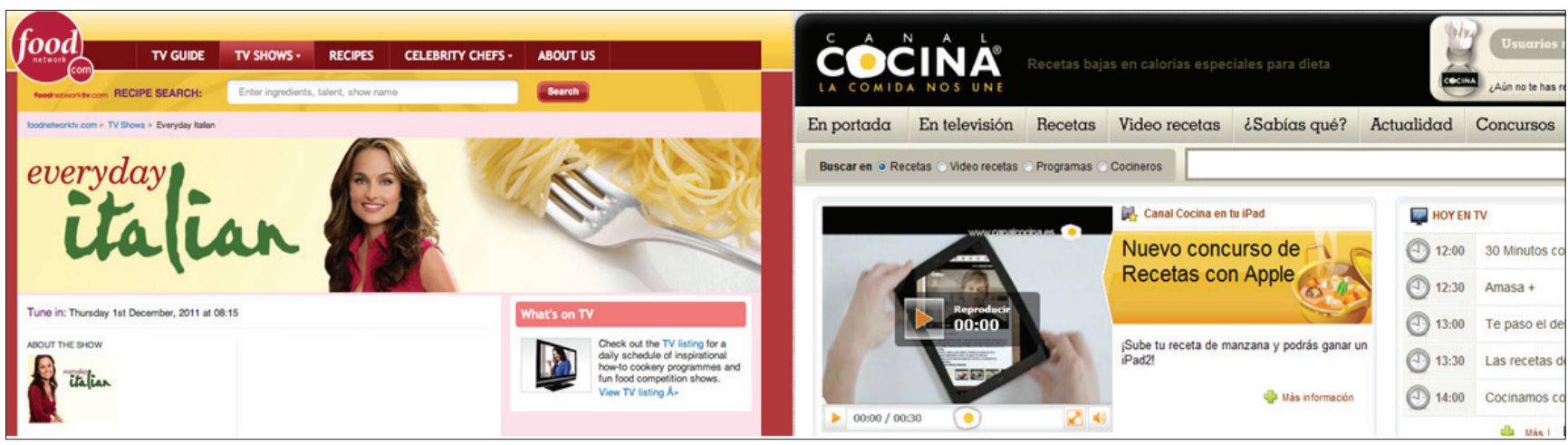

Figura 7. Adecuación al género en canales de TV de cocina. Canal norteamericano con la mujer como protagonista (izquierda) y canal español, mucho más neutral y no estereotipado (derecha)

diendo del objetivo de mercado e influencia socio-cultural, el diseño puede utilizar estereotipos muchos más marcados y distantes (orientación de producto directa) o más equitativos (orientación indirecta o mixta) (figura 7).

\section{Estructura y diseño}

Muchas veces el diseño y la organización de la información dan lugar a dos productos casi distintos según la influencia cultural (Chen et al., 2005; Jagne; Smith-Atakan, 2006). Este rediseño es llevado a cabo en productos donde se necesita mayor asimilación y familiaridad por parte de los usuarios con los contenidos multimedia (figura 8). En el caso de la arquitectura de la información, existen diferencias en cuanto a densidad de información y menú de navegación. Estas tendencias en diseño son fruto del procesamiento cognitivo influenciado por el desarrollo de la escritura. Mientras en la cultura occidental se tiende a la profundidad jerárquica y verticalidad; en culturas asiáticas predomina la organización horizontal menos jerarquizada y con mayor densidad de opciones (figura 9) (Hofstade, 1998; 2005; Abramson et al., 1993; Savvas; El-Kot; Sadler-Smith, 2001; Allinson; Hayes, 2000).

\section{Diseño emocional y experiencia de usuario}

Uno de los aspectos que caracterizan la UX es la respuesta emocional del usuario ante el proceso interactivo, factor que puede provocar deseo u odio por el mismo (Cokcton, 2008; Brave; Nass, 2002). Existen numerosos estudios sobre las emociones en la bibliografía especializada, incluso se han propuesto definiciones consensuadas del término y de sus propiedades:

Una respuesta emocional o sentimiento es una reacción psicológica relacionada con las necesidades, objetivos o preocupaciones de un individuo y que está compuesta por
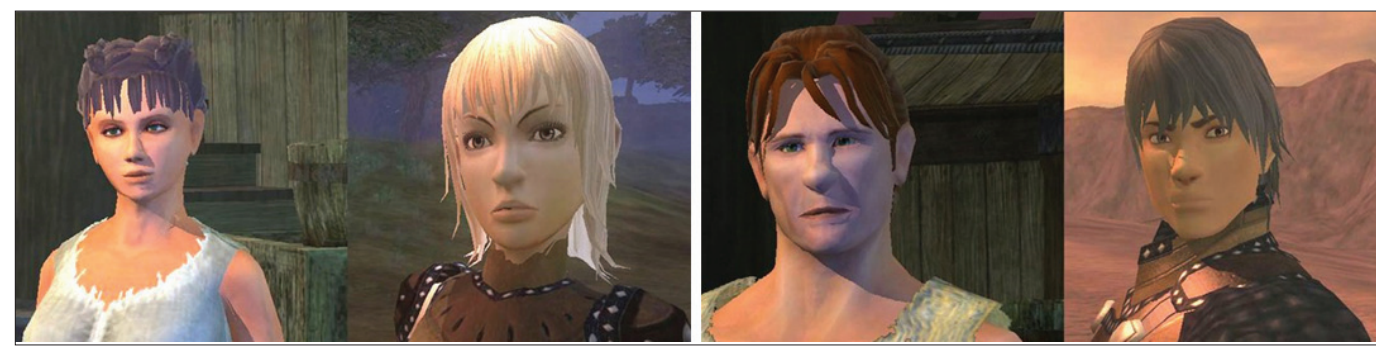

Figura 8. Ejemplos de orientación al mercado en el diseño de un producto con Everquest /l en personajes femeninos y masculinos. Izquierda versión occidental, derecha versión oriental. factores conductuales, fisiológicos, afectivos y cognitivos, en respuesta a unos estímulos que se producen a cuando se interactúa con un producto y son propias de cada individuo (Brave; Nass, 2002; Merhabian, 1994).

Según Norman $(2004 ; 2009)$ las emociones ante el diseño de un producto pueden clasificarse en:

- Visceral: es intuitiva y se debe a la naturaleza del usuario. Se genera de manera automática a través de los sentidos, ya sea visual o auditivamente.

- Conductual: asociada al comportamiento que tiene el usuario con el producto y a la interacción entre ambos. Puede fomentarse a partir de las acciones y consecuencias de éstas durante el proceso de uso.

- Cognitiva: aparece a través de los pensamientos y recuerdos que el usuario tiene al usar un producto. Por lo tanto, es importante manejar sentimientos y situaciones cotidianas que conozca el usuario para afectarle positiva o negativamente.

El uso de las emociones ayuda a obtener una gran experiencia interactiva. Fomentar alegría, presión, frustración, miedo, intriga, curiosidad, atractividad..., ayuda a mejorar la satisfacción del usuario, uno de los aspectos del estándar ISO/ IEC 25010, y favorece una mejor recepción y motivación del usuario para que realice una acción determinada. Norman (2004) y Lazzaro (2008), indican que uno de los secretos de la UX es el manejo de las emociones y, entre ellas, la motivación es clave. Si conseguimos motivar a nuestro usuario por un producto, experimentará todas las facetas que ofrece.

Como las emociones son automáticas y dinámicas, es importante captarlas en el instante en que se producen. Así, podemos mejorar la experiencia del usuario mediante el feedback emocional (Mehrabian, 1994; Desmet; Overbeeke; Tax, 2001), evitando que éste evalúe un sitio web con número o adjetivos que pueden llevarle a confusión. Unos simples emoticonos pueden representar más objetivamente cómo se ha sentido el usuario que una 


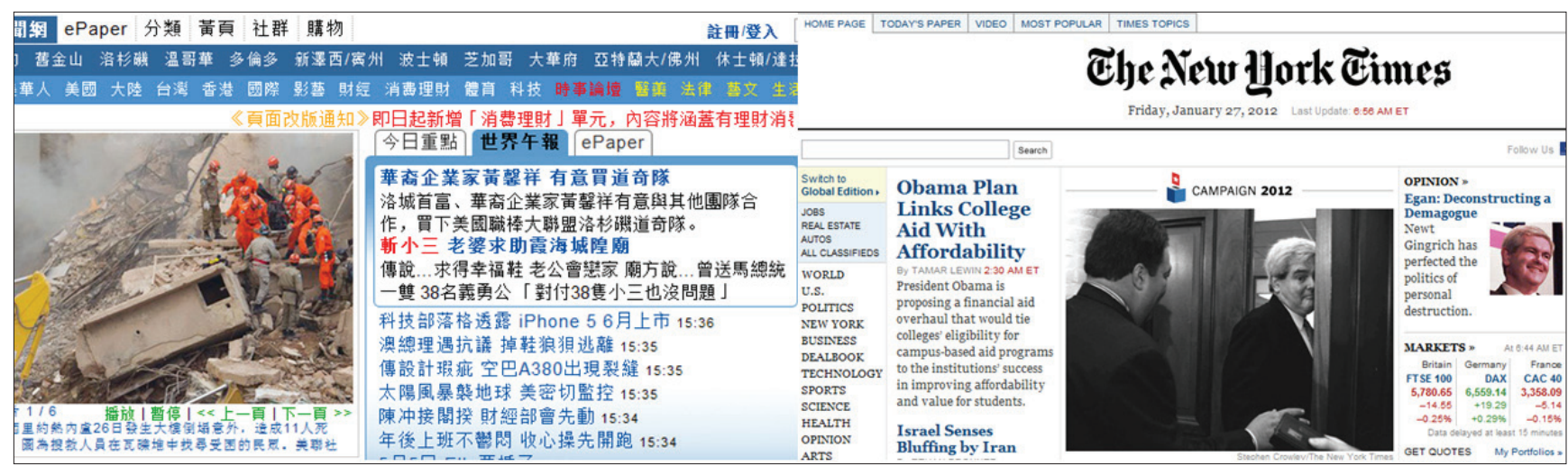

Figura 9. Ejemplos de organización de la información en menús: The world journal (periódico chino: horizontal y denso) y The New York times (estadounidense: vertical y menos apretado)

serie de adjetivos o números que pueden ser más imprecisos (figura 10). Estos métodos ayudan y completan los tradicionales tests y cuestionarios de satisfacción y de calidad en uso como pueden ser los conocidos: CUSQ (Computer usability satisfaction questionnaires) (Lewis, 1995), QUIS (Questionnaire for user interaction satisfaction) (Chin et al., 2008) o SUMI (Software usability measurement inventory) (Kirakowski, 1996).

Hay que conocer el contexto cultural de los usuarios y asegurar que la elección de metáforas, colores y organización es la adecuada

Las webs de contenidos compartidos o redes sociales, se han convertido en grandes exponentes en la generación de información y han promovido una mejora de la experiencia emocional. El diseño y contenido tradicional de la información se ve enriquecido por los comentarios y aportaciones del resto de usuarios que hacen uso del producto. Esta tendencia de participación social en la generación de contenidos es una de las características identificadoras para representar la evolución seguida por la Web, denominándolas web 2.0 (Karr, 2010). Los usuarios se sienten protagonistas en la construcción de la información, siendo estos sistemas su escaparate en la red de redes, participando en debates, siguiendo las actividades de sus contactos. Las redes sociales se han convertido en lugares globales de encuentro e interacción social y en herramientas de gran utilidad para empresas, artistas o marcas, a través de las que intensifican su relación con el usuario para mejorar la aceptación final o experiencia con sus productos. Basta pensar, por ejemplo, en el botón "me gusta" de Facebook o del "+1" de Google+. Además aquí vemos un ejemplo de la importancia de la localización. Mientras Facebook debe traducir su mensaje a diferentes lenguas, Google ha optado por un mensaje internacionalmente entendible para todo tipo de usuarios.

Se están utilizando técnicas en el diseño de productos como la gamificación para conseguir la participación social y ganar seguidores a una marca. Esta técnica consiste en aplicar mecánicas propias de juegos en contextos ajenos a éstos, con el objetivo de "incentivar un determinado comportamiento en el usuario" (Bunchball, 2010). Así conseguimos que los usuarios se sientan motivados por usar el producto ofreciendo algún tipo de recompensa que premie las acciones que realicen en nuestro sitio web (emociones conductuales) o utilizarlas para generar un cambio en el proceso de uso generando comportamientos deseados aprovechándose de la predisposición psicológica de los seres humanos para participar en juegos. Técnicas como la descrita han servido para generar un sentimiento de fidelidad hacia la marca, mejorando la UX. Como ejemplos de su éxito podemos destacar los códigos dados por determinadas compras en productos de Coca-Cola o Starbucks, que al ser introducidos en la web, premiaban a los usuarios con el acceso a nuevas secciones donde poder ganar premios (figura 11). De la misma manera, el juego Assassin's Creed fue pionero en conectar las acciones realizadas en el propio juego (jugado en PC o videocon-
You should find that the system is very user-friendly, and you can get help at any time via the Get Help Now section/FAQ Knowledgebase. If you forget your password, you can enter your email address in the Password Help field and click Go, and the system will send you an email containing instructions for resetting your password. If you are unsure whether you have an account, please do not create a new account. Please contact the journal who will be able to check if you have an account on the site. (If you have difficulty finding the relevant contact please email authorqueries@tandf.co.uk.)

Once your manuscript has been accepted, the transfer to the Production Editor at Taylor \& Francis is faster, with the files and associated information going straight into CATS, our article tracking system, so that it can begin the production process.

Was this page helpful?

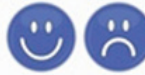

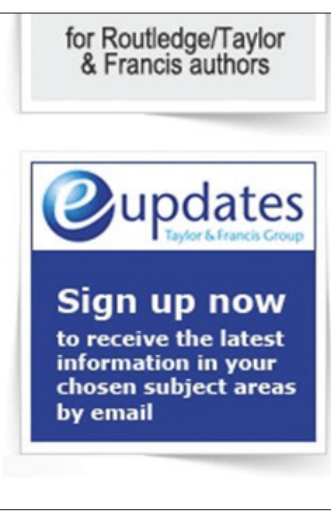

back to top

sola) con los logros y acciones realizados en Facebook. Así logró que los usuarios jugaran al juego incluso cuando no lo hacían, es decir, mientras realizan actividades en la Web y en Facebook, obteniendo premios especiales que luego podían utilizar en el juego de videoconsola o PC, o influyendo en cómo deberían realizar

Figura 10. Uso de feedback emocional en la web de ayuda para autores en Taylor \& Francis 


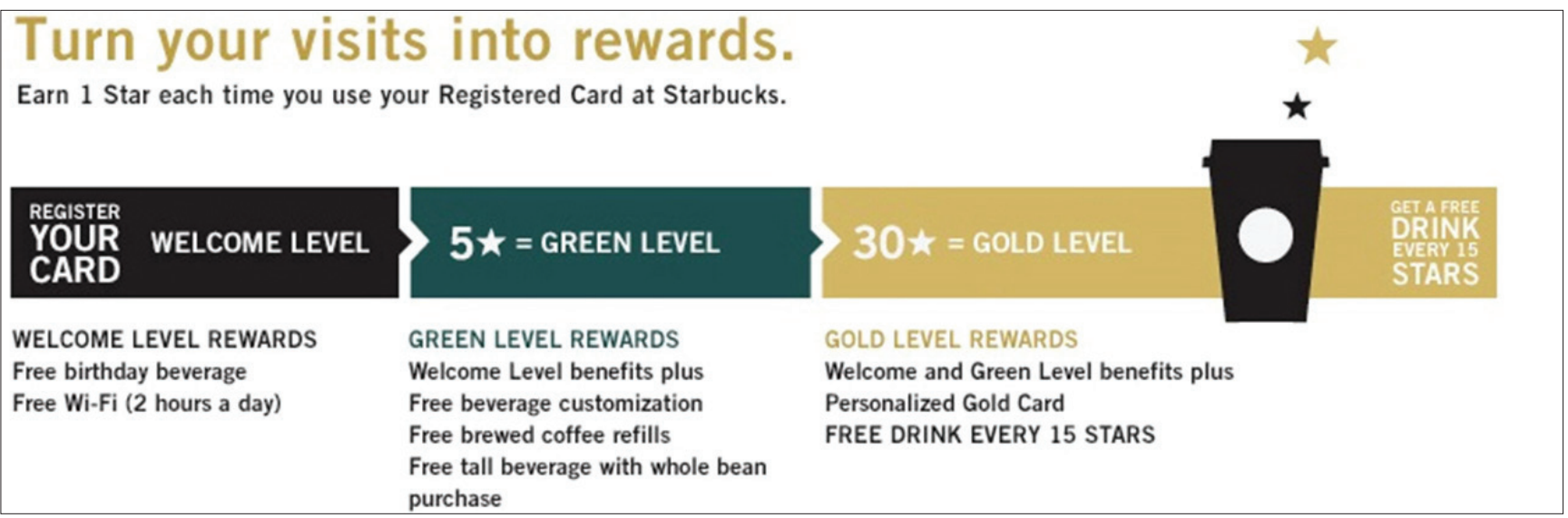

Figura 11. My Starbucks Reward o cómo usar mecánicas de niveles de juegos y desarrollar fidelidad a una marca

distintos aspectos que el juego mostraba (modificación del proceso de uso hacia una actividad deseada), premiando en el juego la visita reiterada en la web y fidelidad de los usuarios hacia el producto y marca.

Los colores, a parte de tener un gran peso estético, ayudan a dotar de significado emocional a la información que estamos mostrando, favoreciendo la aparición de ciertas emociones viscerales (Sharpe, 1974; Birren, 1978; Jacobs; Hustmyer, 1974). La psicología de los colores es muy utilizada en el diseño de productos y debe responder a lo que queramos que el usuario experimente cuando interactúe con nuestro producto o marca (figura 12).

\section{Conclusiones y trabajos futuros}

A lo largo de este artículo nos hemos adentrado en el concepto de la experiencia de usuario como factor determinante para asegurar el éxito de un producto. Día a día, la competencia en el mercado obliga a los diseñadores, arquitectos de información y especialistas a buscar ese factor diferencial que haga ganar cuota en un mercado cada día más saturado y competitivo.

La correcta experiencia interactiva es resultado de un proceso en el que intervienen multitud de factores: individuales, sociales, culturales, contextuales y propios del producto; está influida por experiencias previas que condicionan las expectativas y experiencias futuras. Su análisis hace especial énfasis en factores como son el bagaje cultural y social, el comportamiento emocional del usuario y la estética. Para lograr una buena experiencia en el diseño de productos interactivos o de información es de vital importancia analizar al usuario, para ofrecerle las mejores sensaciones. Factores como los descritos ayudan a identificar nuestro producto y hacerlo único.

Para asegurar la calidad de un producto hay que potenciar factores hedónicos que lo acerquen a las preferencias del usuario

A través de ejemplos comerciales se ha mostrado la aplicación de estos factores. En ellos queda patente la necesidad de ir más allá, fomentar una mejor experiencia hedónica que acerque el producto a las preferencias del usuario final. Intentar esta experiencia hace que el diseño se vea influido por los usuarios en la elección de los colores, lenguaje, metáforas o diseño de la arquitectura de la información. Se han mostrado nuevos estándares ISO que abogan y tienen en cuenta estos elementos como identificadores de la calidad de un producto desarrollado.

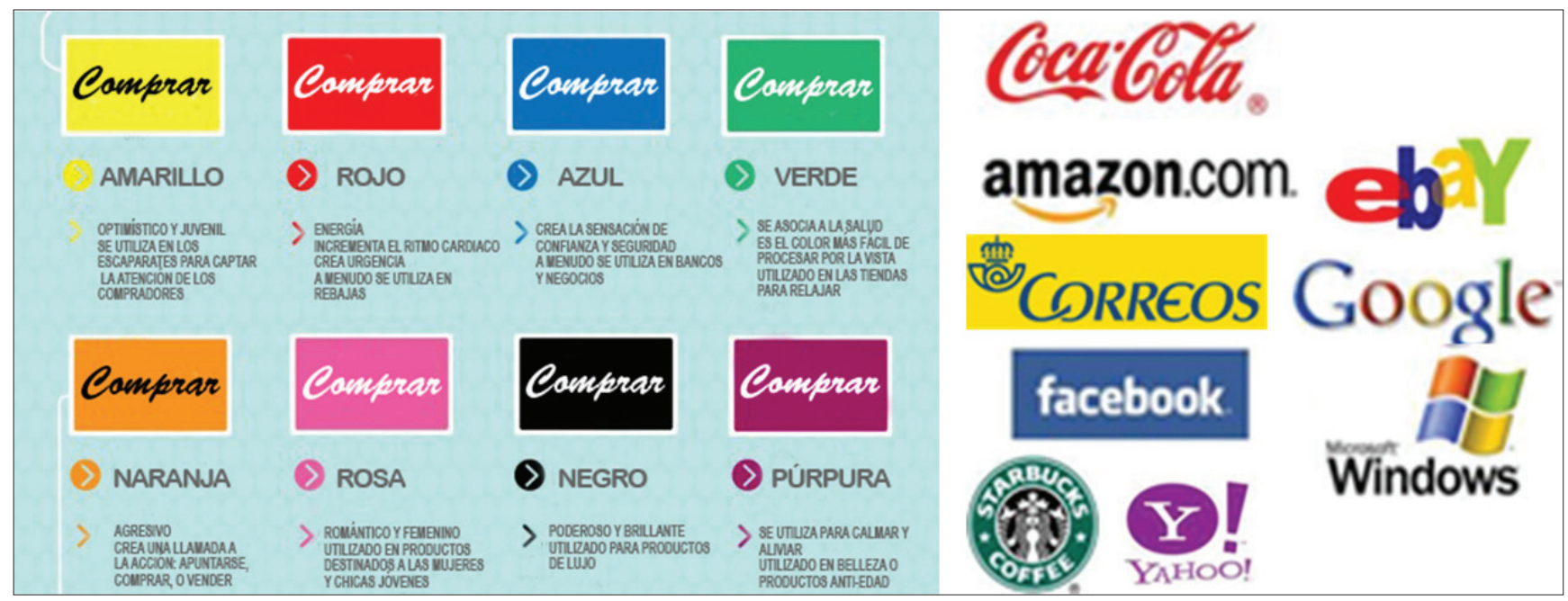

Figura 12. Colores dominantes en espacios web de marcas conocidas y emociones relacionadas 
Como trabajos futuros, se están identificando elementos usados en otros sistemas como videojuegos o aplicaciones móviles con el objetivo de asegurar una correcta calidad en uso que mejore la experiencia interactiva. Además, se estátrabajando en la clasificación de los usuarios a través de modelos mentales o de actuación, en busca de preferencias que sirvan como guías de desarrollo de productos web, con el fin de adaptar su contenido a una determinada cultura, población o sector del mercado al que va dirigido.

\section{Agradecimientos}

Este trabajo está financiado por el Ministerio de Economía y Competitividad de España, a través de los proyectos de investigación OMediaDis (TIN2008-06228) y VIDECO (TIN2011-26928).

\section{Bibliografía}

Abramson, Neil; Lane, Henry; Nagai, Hirohisa; Takagi, Haruo (1993). "A comparison of Canadian and Japanese cognitive styles: implications for management interaction". Journal of international business studies, v. 24, n. 3, pp. 575-587.

Allinson, Christopher W.; Hayes, John (2000). "Cross-national differences in cognitive style: implications for management". The intl journal of human resource management, $\mathrm{v}$. 11, n. 1 , pp. $161-170$.

http://dx.doi.org/10.1080/095851900340042

Andreu-Vall, Mar; Marcos, Mari-Carmen (2011). "Multilingüismo en la Web: pautas para un diseño centrado en el usuario". En: Garay, Néstor; Abascal, Julio (eds.): Actas del XII Congreso intl interacción, pp. 209-212.

http://eprints.rclis.org/bitstream/10760/16407/1/ interaccion_2011_word.pdf

Andreu-Vall, Mar; Marcos, Mari-Carmen (2012). “Evaluación de sitios web multilingües: metodología y herramienta heurísti-ca". El profesional de la información, mayo-junio, v. 21, n. 3, pp. 254-260.

http://dx.doi.org/10.3145/epi.2012.may.05

Baeza-Yates, Ricardo; Rivera-Loaiza, Cuauhtémoc; VelascoMartín, Javier (2004). "Arquitectura de la información y usabilidad en la Web". El profesional de la información, v. 13, n. 3, pp. 169-178.

http://www.mantruc.com/files/art-epi-2004.pdf

Bevan, Nigel (2000). "Quality in use for all”. En: Stephanidis, Constantine; Erlbaum, Lawrence (eds.). User interfaces for all.

Bevan, Nigel (1999). "Quality in use: meeting user needs for quality". Journal of systems and software, v. 49, n. 1, pp. 8996.

http://www.usabilitynet.org/papers/qiuse.pdf

http://dx.doi.org/10.1016/S0164-1212(99)00070-9

Birren, Faber (1978). Color and human response. Van Nostrand Reinhold Co. ISBN: 9780442207878

Brave, Scott; Nass, Clifford (2002). "Emotion in humancomputer interaction". En: Jacko, Julie; Sears, Andrew (eds.). The human-computer interaction handbook: fundamentals, evolving technologies and emerging applications. Lawrence
Erlbaum Associates, pp. 81-96. ISBN: 9780805844689

Bunchball (2010). Gamification 101: an introduction to the use of game dynamics to influence behavior.

http://www.bunchball.com/sites/default/files/downloads/ gamification101.pdf

Chen, Mark; Cuddihy, Elisabeth; Thayer, Alex; Zhou, Quan (2005). "Creating cross-cultural appeal in digital games: issues in localization and user testing". En: 52nd annual conf for the Society for Technical Communication (STC).

Chin, John P.; Diehl, Virginia A.; Norman, Kent (1988). “Development of an instrument measuring user satisfaction of the human-computer interface". En: $\mathrm{CHI}$ '88. Procs. of the Sigchi conf on Human factors in computing systems. Washington DC, pp. 213-218.

http://dx.doi.org/10.1145/57167.57203

Cockton, Gilbert (2008). "Putting value into e-valuation". En: Lai-Chong, Effie; Hvannberg, Ebba; Cokton, Gilbert (eds.). Maturing usability: quality in software, interaction and value. Springer Verlag, pp. 287-317.

http://dx.doi.org/10.1007/978-1-84628-941-5_13

Collazos, César A.; Gil, Rosa (2011). "Using cross-cultural features in Web design patterns". En: Eighth int conf on information technology: new generations (ITNG), IEEE Press, pp. 514-519.

http://doi.ieeecomputersociety.org/10.1109/ITNG.2011.95

Desmet, Pieter; Overbeeke, Kees; Tax, Stefan (2001). “Designing products with added emotional value: development and application of an approach for research through design". The design journal, v. 4, n. 1, pp. 32-47.

http://dx.doi.org/10.2752/146069201789378496

Garrett, Jesse-James (2010). The elements of user experience: user-centered design for the Web and beyond (2nd edition). New Riders Press. ISBN: 9780321683687

Hassan-Montero, Yusef; Martín-Fernández, Francisco J. (2005). "La experiencia del usuario". No sólo usabilidad, n. 4. http://www.nosolousabilidad.com/articulos/experiencia_ del_usuario.htm

Hassenzahl, Marc (2003). "The thing and I: understanding the relationship between user and product". En: Blythe, Mark; Monk, Andrew; Overbeeke, Kees; Wright, Peter (eds.). Funology: from usability to enjoyment. Dordrecht: Kluwer Academic Publishers, pp. 31-42.

http://www.uni-landau.de/hassenzahl/pdfs/03\%20 Hassenzahl.pdf

Hofstede, Geert (2005). Cultures and organizations: software of the mind. New York, NY, USA. ISBN: 9780071439596

Hofstede, Geert (1980). Culture's consequences: international differences in work-related values. Newbury Park, CA: Sage. ISBN: 9780803913066

Hofstede, Geert (1998). Masculinity and femininity: the taboo dimension of national cultures. An introduction and consequences for gender roles, sexual behavior and religion. ISBN: 9780761910282

ISO 9241-11:1998 (1998). Ergonomic requirements for office 
work with visual display terminals (VDTs). Part 11: Guidance on usability.

http://www.it.uu.se/edu/course/homepage/acsd/vt09/ ISO9241part11.pdf

ISO/IEC 9126:1991 (1991). Software engineering. Product quality.

ISO/IEC 9126-1:2001 (2001). Software engineering. Product quality. Part 1: Quality model.

ISO/IEC 9241-210:2010 (2010). Ergonomics of human-system interaction. Part 210: Human centred design for interactive systems. Clausule 2.15.

ISO/IEC 25010:2011 (2011). Systems and software engineering. Systems and software quality requirements and evaluation (SQUaRE). System and software quality models.

Jacobs, Keith W.; Hustmyer, Frank (1974) "Effects of four psychological primary colors on GSR, heart rate and respiration rate". Percept mot skills, 1974, v. 38, n. 3, pp. 763-766. http://www.amsciepub.com/doi/abs/10.2466/pms.1974.38.3.763

Jagne, Jainaba; Smith-Atakan, A. Serengul (2006). "Crosscultural interface design strategy". Universal access in the information society, v. 5, n. 3, pp. 299-305.

http://dx.doi.org/0.1007/s10209-006-0048-6

Karr, Douglas (2008). I believe in web 3.0!.

http://www.marketingtechblog.com/web-1-web-2-web-3-0

Kirakowski, Jurek (1996). "The software usability measurement inventory: Background and usage". En: Jordan, Patrick; Thomas, Bruce; Weerdmeester, Bernard; McClelland, Ian (eds.). Usability evaluation in industry. UK: Taylor and Francis, pp. 169-178. ISBN: 0748404600

Krug, Steve (2005). Don't make me think: a common sense approach to Web usability, 2nd Edition. New Riders Press. ISBN: 9780321344755

Law, Effie Lai-Chong (2011). "The measurability and predictability of user experience". En: Procs of the 3rd ACM Sigchi symposium on engineering interactive computing systems (EICS'11), pp. 1-10. ISBN: 9781450306706

http://dx.doi.org/10.1145/1996461.1996485

Law, Effie Lai-Chong; Roto, Virpi; Hassenzahl, Marc; Vermeeren, Arnold; Kort, Joke (2008). "Towards a shared definition of user experience". En: Procs of CHI EA'08, (extended abstracts on human factors in computing systems pages). New York, pp. 2395-2398.

http://dx.doi.org/10.1145/1358628.1358693

Law, Effie Lai-Chong; Roto, Virpi; Hassenzahl, Marc; Vermeeren, Arnold; Kort, Joke (2009). "Understanding, scoping and defining user experience: a survey approach". En: Procs of CHII2009. User experience. Boston, MA, pp. 719-728. http://www.itu.dk/ jeppeh/specialeartikler/Understanding, Scoping and Defining User eXperience.pdf

Law, Effie Lai-Chong; Van-Schaik, Paul (2010). “Modeling user experience - An agenda for research and practice". Interacting with computers, v. 22, n. 5, pp. 313-322.

http://dx.doi.org/10.1016/j.intcom.2010.04.006

Lazzaro, Nicole (2008). "The four fun keys". En: Isbister, Katherine; Schaffer, Noah (eds.). Game usability: advancing the player experience. Morgan Kaufmann. ISBN: 978 0123744470

Lewis, James R. (1995). “IBM computer usability satisfaction questionnaires: psychometric evaluation and instructions for use". Intl journal of human-computer interaction, v. 7, n. 1, pp. 57-78. http://dx.doi.org/10.1080/10447319509526110

LLC (2009). The cultural iceberg. http://www.languageandculture.com/cultural-iceberg

Maslow, Abraham H. (1943). "A theory of human motivation". Psychological review, v. 50, n. 4, pp. 370-396. http://dx.doi.org/10.1037/h0054346

Mayhew, Deborah J. (1999). The usability engineering lifecycle: a practitioner's handbook for user interface design (interactive technologies). Morgan Kaufmann. ISBN: 978 1558605619

McCandless, David (2010). Information is beautiful. Collins. ISBN: 9780007294664

Mehrabian, Albert (1994). Manual for the revised trait arousability (converse of the stimulus screening). Alta Mesa Road, Monterey, CA, USA.

Nielsen, Jacob (1993). Usability engineering, 1st ed. Morgan Kaufmann. ISBN: 9780125184069

Norman, Donald A. (2004). Emotional design: why we love (or hate) everyday things. New York: Basic Books. ISBN: 978 0465051359

Norman, Donald A. (2009). The design of future things. New York: Basic Books. ISBN: 9789465002277

Sharpe, Deborah T. (1974). The psychology of color and design. Nelson-Hall Co. ISBN: 9780882291079

Savvas, Michael; El-Kot, Ghada; Sadler-Smith, Eugene (2001). "Comparative study of cognitive styles in Egypt, Greece, Hong Kong and the UK". Intl journal of training and development, v. 5, n. 1, pp. 64-73.

http://dx.doi.org/10.1111/1468-2419.00122

Shen, Siu-Tsen; Woolley, Martin; Prior, Stephen (2006). "Towards culture-centred design". Interacting with computers, v. 18, n. 4, pp. 820-852.

http://dx.doi.org/10.1016/j.intcom.2005.11.014

Vermeeren, Arnold P.; Law, Effie Lai-Chong; Roto, Virpi; Obrist, Marianna; Hoonhout, Jettie; Väänänen-Vainio-Mattila, Kaisa (2010). "User experience evaluation methods: current state and development needs". En: Procs of the 6 th Nordic conf on human-computer interaction extending boundaries, NordiCHI2011, pp. 521-530. http://dx.doi.org/10.1145/1868914.1868973 